\title{
Modeling of the formation of diamond-like phases from structural varieties of tetragonal graphite
}

\author{
V. A. Greshnyakov, E. A. Belenkov ${ }^{\dagger}$ \\ †belenkov@csu.ru
}

Chelyabinsk State University, 129 Bratiev Kashirinykh st., 454001, Chelyabinsk, Russia

\begin{abstract}
Theoretical investigations of the phase transformations of structural modifications of tetragonal $\mathrm{L}_{4-8}$ graphite in diamond-like phases were performed using the density functional theory method in local density approximation (LDA) and generalized gradient approximation (GGA). The varieties of $\mathrm{L}_{4-8}$ graphite were examined with the following packing of graphene layers: AA $(P 4 / \mathrm{mmm}), \mathrm{AB}(\mathrm{I} 4 / \mathrm{mmm}), \mathrm{ABCD}\left(\mathrm{I}_{1} / \mathrm{amd}\right)$. The LA6 (Imma), LA7 ( $\left.\mathrm{Cmcm}\right)$, and LA10 (I4 / amd) diamond-like phases can be obtained from these graphites under compression. The LA6 diamond-like phase can be obtained from $\mathrm{L}_{4-8} \mathrm{AA}_{\mathrm{Araphite}}$ at pressure of $44 \mathrm{GPa}$. Another phase, LA7, can be formed from tetragonal $\mathrm{L}_{4-8} \mathrm{AB}$ graphite in the pressure range from 43 to $46 \mathrm{GPa}$. The LA10 structure can be obtained only from $\mathrm{L}_{4-8}$ graphite with the ABCD layer packing of at pressures from 32 to $40 \mathrm{GPa}$. It was established that « $\mathrm{L}_{4-8}$ AA graphite $\rightarrow$ LA6 phase», « $\mathrm{L}_{4-8}$ AB graphite $\rightarrow$ LA7 phase», and « $\mathrm{L}_{4-8}$ ABCD graphite $\rightarrow$ LA10 phase» structural transitions are exothermic phase transitions of the first kind, as a result of which an energy of $0.5,0.5$, and $0.3 \mathrm{eV} /$ atom is released, respectively. The energy barriers that need to be overcome to observe reverse «LA6 $\rightarrow$ $\mathrm{L}_{4-8} \mathrm{AA}$ graphite», «LA7 $\rightarrow \mathrm{L}_{4-8} \mathrm{AB}$ graphite» $и$ «LA10 $\rightarrow \mathrm{L}_{4-8} \mathrm{ABCD}$ graphite» phase transitions are $0.38,0.34$, and $0.18 \mathrm{eV} /$ atom for the LDA-calculations or $0.31,0.28$, and $0.13 \mathrm{eV} /$ atom for the GGA-calculations, respectively. Possible ways of synthesizing LA6, LA7, and LA10 phases are a strong compression of graphite along the [001]-axis at low temperatures.
\end{abstract}

Keywords: diamond-like phase, tetragonal graphene, phase transition.

\section{Моделирование процесса формирования алмазоподобных фаз из структурных разновидностей тетрагонального графита}

\author{
Грешняков В. А., Беленков Е. А. ${ }^{\dagger}$ \\ tbelenkov@csu.ru
}

Челябинский государственный университет, ул. Братьев Кашириных 129, 454001, Челябинск, Россия

В данной работе выполнено теоретическое исследование фазовых превращений различных структурных модификаций тетрагонального графита $\mathrm{L}_{4-8}$ в алмазоподобные фазы при использовании метода теории функционала плотности в приближении локальной плотности (ПЛП) и обобщенном градиентном приближении (ОГП). Были рассмотрены основные разновидности графита $\mathrm{L}_{4-8}$ со следующей упаковкой графеновых слоев: АА (пространственная группа $P 4 / \mathrm{mmm}), \mathrm{AB}(\mathrm{I} / \mathrm{mmm})$ и $\mathrm{ABCD}\left(\mathrm{I}_{1} / \mathrm{amd}\right)$. Анализ показал, что из этих графитов при сжатии могут быть получены алмазоподобные фазы LA6 (Imma), LA7 ( Cmcm) и LA10 (I4/ / amd). Установлено, что алмазоподобная фаза LA6 может быть получена из графита $\mathrm{L}_{4-8}$ AA при давлении 44 ГПа. Фаза LA7 может быть сформирована из тетрагонального графита $\mathrm{L}_{4-8} \mathrm{AB}$ в диапазоне давлений от 43 до 46 ГПа. Структура алмазоподобной фазы LA10 может быть получена только из графита $\mathrm{L}_{4-8}$ с упаковкой слоев $\mathrm{ABCD}$ в области давлений от 32 до 40 ГПа. Расчеты показали, что структурные переходы «графит $\mathrm{L}_{4-8} \mathrm{AA} \rightarrow$ фаза LA6», «графит $\mathrm{L}_{4-8} \mathrm{AB} \rightarrow$ фаза LA7» и «графит $\mathrm{L}_{4-8} \mathrm{ABCD} \rightarrow$ фаза LA10» являются экзотермическими фазовыми переходами первого рода, в результате которых выделяется энергия $0.5,0.5$ и 0.3 эВ/атом, соответственно. Энергетические барьеры, которые необходимо преодолеть для наблюдения обратных фазовых переходов «LA6 $\rightarrow$ графит $\mathrm{L}_{4-8} \mathrm{AA}$ », «LA7 $\rightarrow$ графит $\mathrm{L}_{4-8} \mathrm{AB}$ » и «LA10 $\rightarrow$ графит $\mathrm{L}_{4-8} \mathrm{ABCD}$ », составляют 0.38, 0.34 и 0.18 эВ/атом (ПЛП-расчеты) или 0.31, 0.28 и 0.13 эВ/атом (ОГП-расчеты), соответственно. Возможный способ синтеза фаз LA6, LA7 и LA10 - сильное сжатие графита $\mathrm{L}_{4-8}$ вдоль кристаллографической оси [001] при низких температурах.

Ключевые слова: алмазоподобная фаза, тетрагональный графен, фазовый переход. 


\section{1. Введение}

Структурные модификации различных соединений могут относиться к аллотропным разновидностям, полиморфам или политипам [1-3]. Аллотропами углерода являются карбин, графит и алмаз. Каждый из аллотропов имеет ряд полиморфных разновидностей [2]. Полиморфы алмаза - это алмазоподобные материалы, которые состоят из $\mathrm{sp}^{3}$-гибридизированных атомов [4-7]. Такая гибридизация обуславливает наличие трехмерной, ковалентно связанной структуры этих материалов и их высокие механические характеристики. Большинство новых алмазоподобных фаз до настоящего времени являются только теоретически предсказанными [4-7]. Поэтому необходимы исследования, связанные с поиском способов их синтеза. В первую очередь необходимо найти методы получения алмазоподобных фаз, которые согласно теоретическим прогнозам должны быть наиболее устойчивыми. Алмазоподобные фазы могут быть получены из наноструктур-предшественников, таких как фуллереноподобные кластеры, углеродные нанотрубки и графеновые слои $[2,8,9]$. Одними из самых перспективных материалов являются материалы со структурами фаз LA6, LA7 и LA10 [5]. В работах $[4,5,10]$ предложены способы синтеза этих фаз в результате сильного одноосного сжатия новой разновидности графита - тетрагонального графита $\mathrm{L}_{4-8}$. Оценить возможность реализации этого способа на практике можно на основе теоретических расчетов, которые были проведены в данной работе.

\section{2. Методика расчетов}

Расчеты кристаллических структур и энергетических характеристик углеродных фаз выполнены методом теории функционала плотности (DFT) для приближения локальной плотности (LDA) [11] и обобщенного градиентного приближения (GGA) [12]. Влияние ионных остовов учитывалось через сохраняющие норму псевдопотенциалы. Для расчетов были использованы сетки $12 \times 12 \times 12$ из $k$-точек в обратных элементарных ячейках. Волновые функции раскладывались по усеченному базисному набору плоских волн. Для ограничения размерности набора базисных функций значение $E_{\text {cutoff }}$ было принято равным 60 Ридберг.

Модельные исследования структурных преобразований различных модификаций тетрагонального графита $\mathrm{L}_{4-8}$ в алмазоподобные фазы были выполнены по методике, подробно описанной в работах $[13,14]$. Для структурных преобразований между графитоподобными и алмазоподобными фазами производилось сжатие и/или растяжение их структур вдоль направлений, по которым наблюдается наилучшее соответствие мотивов кристаллических решеток этих фаз.

\section{3. Результаты и обсуждение}

Анализ возможных путей формирования двух орторомбических фаз LA6 и LA7 показал, что их структуры могут быть получены из тетрагональных графитов $\mathrm{L}_{4-8}$ с чередованием графеновых слоев $\mathrm{AA}$ и $\mathrm{AB}$, соответственно (рис. 1). В свою очередь, алмазоподобная фаза LA10 может быть сформирована только из графита $\mathrm{L}_{4-8}$ с упаковкой слоев $\mathrm{ABCD}$ (рис. 1). Формирование структуры рассматриваемых алмазоподобных фаз возможно при сжатии графитов вдоль осей [001].

Результаты DFT-LDA и DFT-GGA расчетов структурных параметров и полных энергий различных модификаций графита $\mathrm{L}_{4-8}$ и алмазоподобных фаз приведены в таблице 1.

В процессе моделирования фазовых переходов изменялись значения параметров с элементарных ячеек тетрагональногографита $\mathrm{L}_{4-8}$ супаковками $\mathrm{AA}, \mathrm{AB}$ и $\mathrm{ABCDв}$ виапазонах от 4.853 (4.951) до 6.163 (7.245) $\AA$, от 4.776 (4.890) до 6.163 (7.245) $\AA$ и от 9.896 (9.955) до 12.30 (14.11) $\AA$ соответственно (в скобках указаны величины для метода DFT-GGA). При этом, также изменялись параметры элементарных ячеек фаз LA6, LA7 и LA10: $b$ - от 4.673 (4.733) до 5.943 (5.916) $\AA$, $a$ - от 4.548 (4.596) до 5.784 (5.745) $\AA$, $c$ - от 7.902 (8.008) до 9.474 (9.386) $\AA$, соответственно. Поскольку полные энергии одних и тех же соединений,
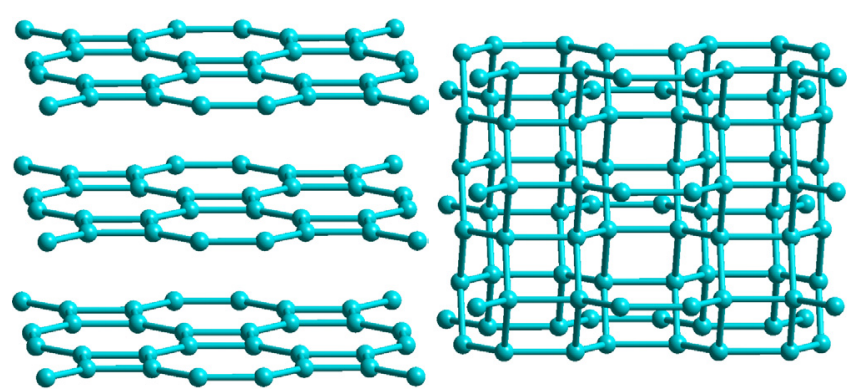

a
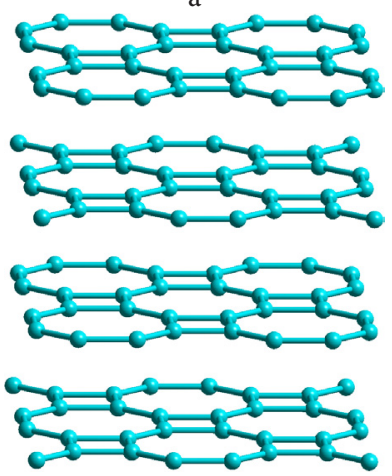

$\mathrm{b}$

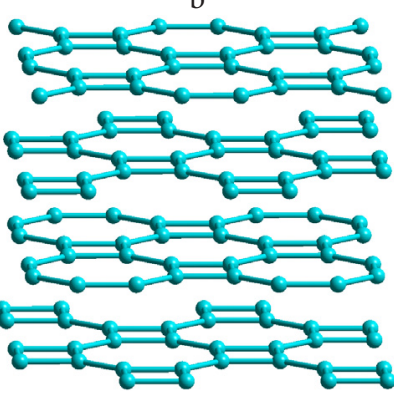

c d

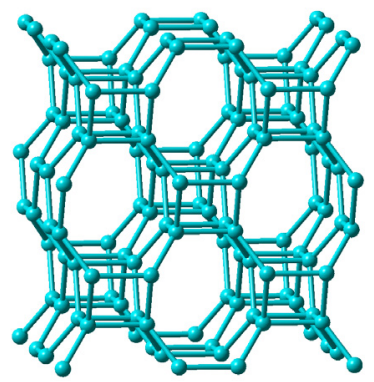

e

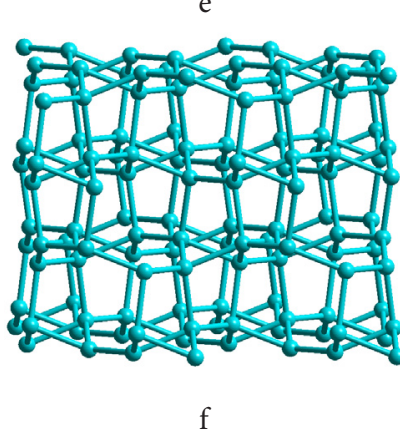

Рис. 1. Кристаллические структуры тетрагональных графитов $\mathrm{L}_{4-8} \mathrm{AA}(\mathrm{a}), \mathrm{AB}(\mathrm{b})$ и $\mathrm{ABCD}(\mathrm{c})$, а также сформированные из них алмазоподобные фазы LA6 (d), LA7 (e) и LA10 (f).

Fig. 1. Crystalline structures of tetragonal $\mathrm{L}_{4-8}$ graphites $\mathrm{AA}$ (a), $\mathrm{AB}(\mathrm{b})$, and ABCD (c), and also LA6 (d), LA7 (e) and LA10 (f) phases. 
рассчитанные в разных приближениях, отличаются на величину $\sim 0.4-0.5$ эВ/атом (таблица 1 ), то для удобства сопоставления фазовых переходов в дальнейшем рассчитывались только разностные полные энергии $\left(\Delta E_{\text {total }}\right)$ относительно наименьшего значения полной энергии.

B результате выполненных DFT-расчетов были построены графики зависимости разностной полной энергии от атомарного объема $\left(V_{a t}\right)$ для прямых и обратных фазовых переходов «графит $\mathrm{L}_{4-8} \mathrm{AA} \leftrightarrow$ фаза LA6» (рис. 2a), «графит $\mathrm{L}_{4-8} \mathrm{AB} \leftrightarrow$ фаза LA7» (рис. 3a) и «графит $\mathrm{L}_{4-8} \mathrm{ABCD} \leftrightarrow$ фаза LA10» (рис. 4a). С помощью данных графиков можно определить энергетические барьеры, которые необходимо преодолеть для структурных переходов между соединениями из $\mathrm{sp}^{2}$ - и $\mathrm{sp}^{3}$-гибридизированных атомов. Значения этих барьеров для прямых $\left(\Delta E_{\mathrm{G} \rightarrow \mathrm{D}}\right)$ и обратных $\left(\Delta E_{\mathrm{D} \rightarrow \mathrm{G}}\right)$ структурных преобразований графита $\mathrm{L}_{4-8} \mathrm{AA}$ в алмазоподобную фазу LA6, рассчитанные методами DFT-LDA и DFT-GGA, составляют $0.16(0.23)$ и 0.38 (0.31) эВ/атом, соответственно (рис. 2a) (в скобках указаны значения для DFT-GGA метода). Фа- зовые переходы между LA7 и графитом $\mathrm{L}_{4-8}$ с упаковкой слоев $\mathrm{AB}$ возможны в результате увеличения полных энергий исходных фаз на $\Delta E_{\mathrm{G} \rightarrow \mathrm{D}}=0.18(0.25)$ эВ/атом и $\Delta E_{\mathrm{D} \rightarrow \mathrm{G}}=0.34(0.28)$ эВ/атом (рис. 3а). И, наконец, фазовые переходы между фазой LA10 и графитом $\mathrm{L}_{4-8}$ $\mathrm{ABCD}$ могут происходить при преодолении следующих энергетических барьеров: $\Delta E_{\mathrm{G} \rightarrow \mathrm{D}}=0.11(0.19)$ эВ/атом, $\Delta E_{\mathrm{D} \rightarrow \mathrm{G}}=0.18(0.13)$ эВ/атом (рис. $\left.4 \mathrm{a}\right)$.

Для прямого фазового перехода графита $\mathrm{L}_{4-8} \mathrm{AA}$ в фазу LA6 давление должно достигать 44.2 (43.9) ГПа, когда объем системы уменьшается до 7.23 (7.46) $\AA^{3} /$ атом (рис. 2a). Структурный переход графита $\mathrm{L}_{4-8} \mathrm{AB}$ в фазу LA7 возможен при достижении атомарного объема 7.17 (7.37) $\AA^{3} /$ атом (рис. 3а) и давления 42.5 (46.3) ГПа. Давление фазового перехода графита $\mathrm{L}_{4-8} \mathrm{ABCD}$ в фазу LA10 составляет 32.3 (39.8) ГПа, причем атомный объем графита в точке структурного преобразования равен 7.34 (7.49) $\AA^{3} /$ атом (рис. 4a).

Все структурные переходы разновидностей тетрагонального графита $\mathrm{L}_{4-8}$ графита в алмазоподобные фазы,

Табл. 1. Параметры элементарных ячеек и полные энергии углеродных соединений (в скобках указаны значения, полученные с помощью метода DFT-GGA).

Table 1. Unit cell parameters and total energies of carbon compounds (the values obtained by the DFT-GGA method are indicated in parentheses).

\begin{tabular}{|c|c|c|c|c|c|c|}
\hline $\begin{array}{l}\text { Фаза } \\
\text { Phase }\end{array}$ & $\begin{array}{l}\text { Пр. группа } \\
\text { Space group }\end{array}$ & $a, \AA$ & $b, \AA$ & $c, \AA$ & $\begin{array}{l}Z \text {, aт. } \\
Z \text {, at. }\end{array}$ & $\begin{array}{l}E_{\text {total, }}, \text { эB/атом } \\
E_{\text {total }}, \mathrm{eV} / \text { atom }\end{array}$ \\
\hline $\mathrm{L}_{4-8} \mathrm{AA}$ & $\mathrm{P} 4 / \mathrm{mmm}$ & $4.867(4.905)$ & $4.867(4.905)$ & $6.010(7.247)$ & 16 & $-157.18(-156.78)$ \\
\hline $\mathrm{L}_{4-8} \mathrm{AB}$ & $I 4 / \mathrm{mmm}$ & $4.867(4.905)$ & $4.867(4.905)$ & $6.008(7.245)$ & 16 & $-157.18(-156.78)$ \\
\hline $\mathrm{L}_{4-8} \mathrm{ABCD}$ & $I 4_{1} / a m d$ & $3.442(3.469)$ & $3.442(3.469)$ & $12.18(13.97)$ & 16 & $-157.19(-156.78)$ \\
\hline LA6 & Imma & $4.900(4.948)$ & $5.079(5.144)$ & $4.176(4.225)$ & 16 & $-157.40(-156.86)$ \\
\hline LA7 & $\mathrm{Cmcm}$ & $4.944(4.995)$ & $4.807(4.869)$ & $4.391(4.442)$ & 16 & $-157.35(-156.81)$ \\
\hline LA10 & $I 4_{1} /$ amd & $3.545(3.581)$ & $3.545(3.581)$ & $8.497(8.611)$ & 16 & $-157.26(-156.73)$ \\
\hline
\end{tabular}

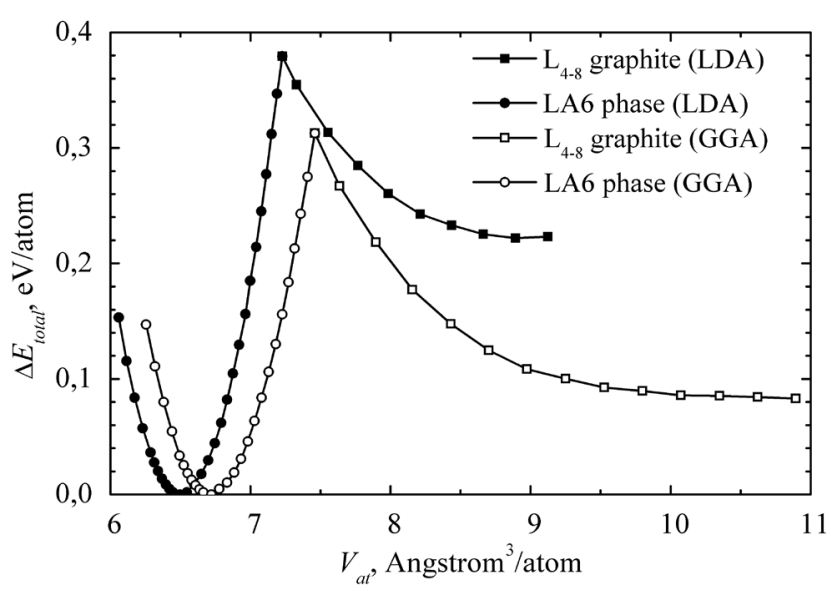

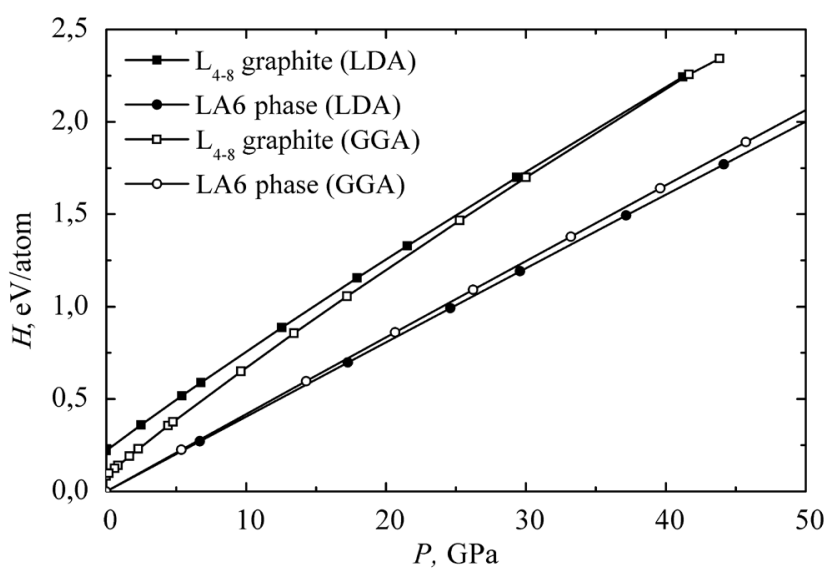

Рис. 2. Графики зависимостей разностной полной энергии от атомарного объема (а) и энтальпии от давления (b) для кристалла графена $\mathrm{L}_{4-8}$ АA и фазы LA6.

Fig. 2. Dependences of the difference total energy on the atomic volume (a) and the enthalpy on the pressure (b) for $\mathrm{L}_{4-8} \mathrm{AA}$ graphite crystal and LA6 phase. 


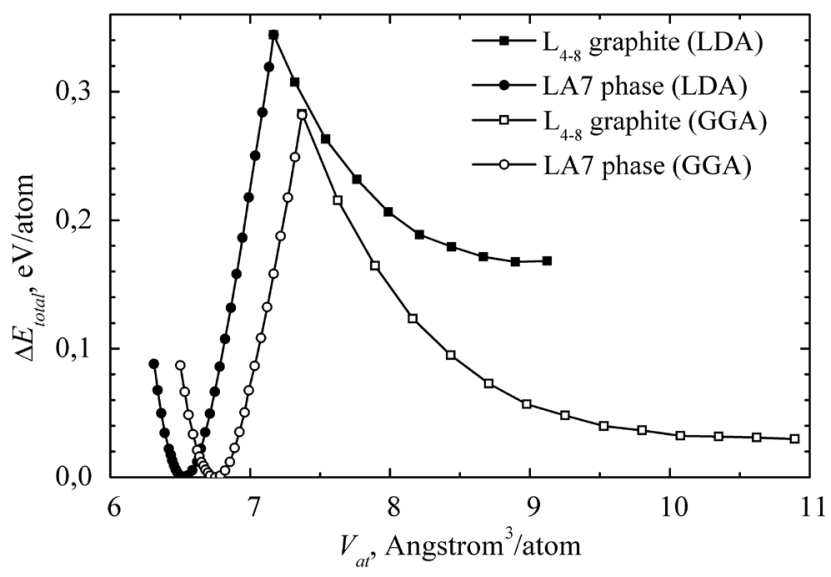

a

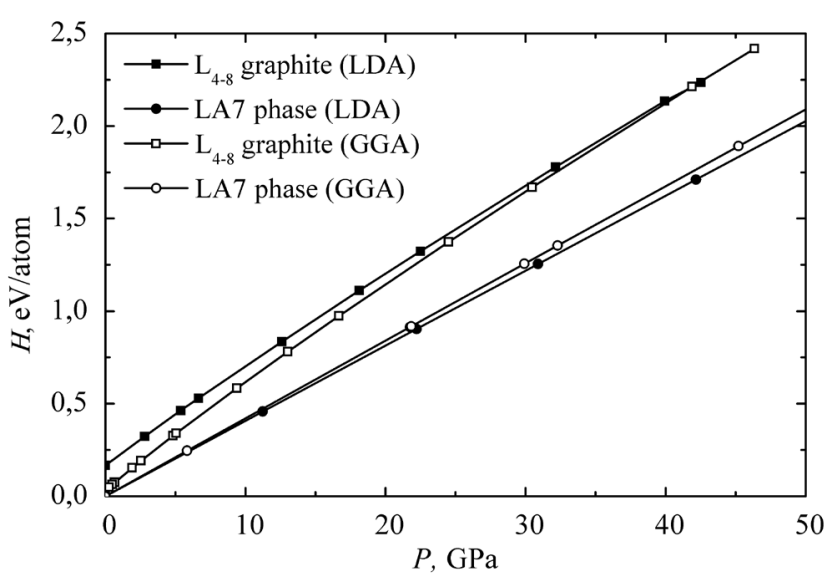

b

Рис. 3. Зависимости $\Delta E_{\text {total }}=f\left(V_{a t}\right)$ (а) и $H=f(P)$ (b) для кристалла графена $\mathrm{L}_{4-8}$ AB и фазы LA7.

Fig. 3. Dependences of $\Delta E_{\text {total }}=f\left(V_{a t}\right)$ (a) and $H=f(P)$ (b) for $\mathrm{L}_{4-8}$ AB graphite crystal and LA7 phase.

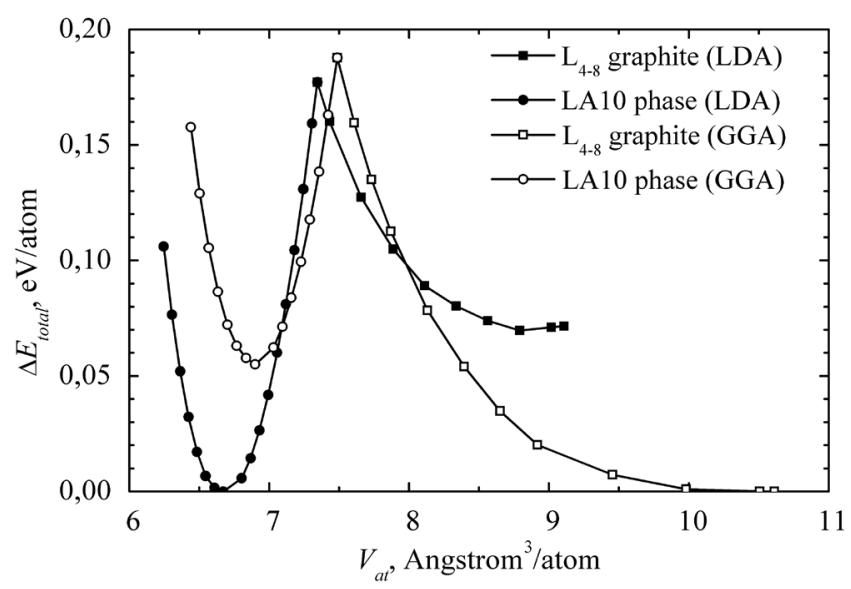

a

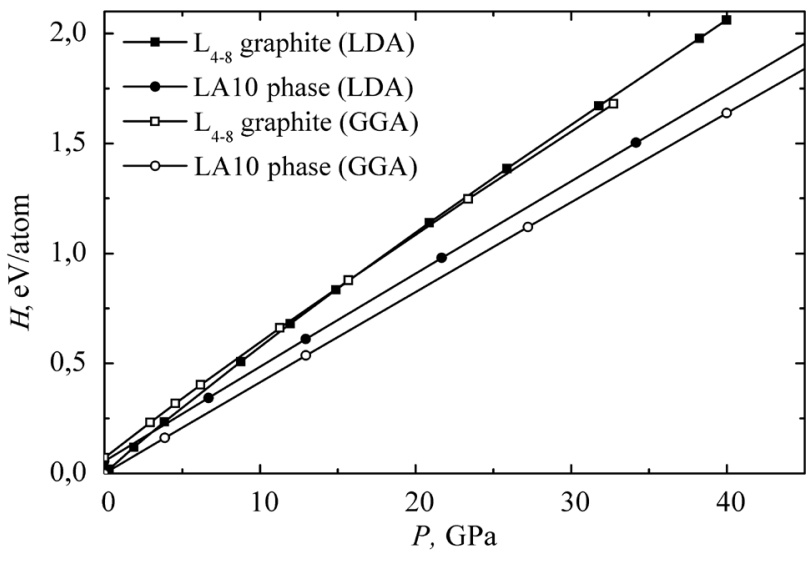

b

Рис. 4. Зависимости $\Delta E_{\text {total }}$ от $V_{a t}$ (а) и $H$ от $P\left(\right.$ b) для графита $\mathrm{L}_{4-8} \mathrm{ABCD}$ и фазы LA10.

Fig. 4. Dependences of $\Delta E_{\text {total }}$ from $V_{a t}$ (a) and $H$ from $P(\mathrm{~b})$ for $\mathrm{L}_{4-8}$ graphite ABCD and LA10 phase.

рассмотренные в данной работе, являются фазовыми переходами первого рода, в процессе которых происходит значительное увеличение плотности на 9.9-12.6\%. Для определения энергии, которая может быть выделена или поглощена при прямом фазовом переходе, были рассчитаны разности энтальпий алмазоподобных фаз и графитов при давлении перехода $\left(\Delta H=H_{\mathrm{D}}-H_{\mathrm{G}}\right)$. Графики зависимостей энтальпии от давления для этих углеродных фаз приведены на рис. $2 \mathrm{~b}, 3 \mathrm{~b}$ и 4b. Установлено, что все прямые фазовые переходы должны иметь экзотермический характер. В результате расчетов были определены следующие значения разности энтальпий: $\Delta H_{\mathrm{L} 4-8 \rightarrow \mathrm{LA} 6}=-0.59(-0.54)$ эВ/атом, $\Delta H_{\mathrm{L} 4-8 \rightarrow \mathrm{LA} 7}=-0.52$ $(-0.48)$ эВ/атом и $\Delta H_{\mathrm{L} 4-8 \rightarrow \mathrm{LA} 10}=-0.33(-0.31)$ эВ/атом $)$.

\section{4. Заключение}

В данной работе методами DFT-LDA и DFT-GGA впервые выполнено теоретическое исследование путей синтеза алмазоподобных фаз LA6, LA7 и LA10. Установлено, что эти алмазоподобные фазы могут быть получены из различных структурных модификаций тетрагональ- ного графита $\mathrm{L}_{4-8}$. Наиболее вероятный способ экспериментального получения фазы LA6 заключается в сильном одноосном сжатии графита $\mathrm{L}_{4-8}$ с упаковкой слоев АА при давлении 44 ГПа. В свою очередь, алмазоподобная фаза LA7 может быть сформирована из графита $\mathrm{L}_{4-8}$ с упаковкой слоев $\mathrm{AB}$ в диапазоне давлений от 43 до 46 ГПа. Последняя из рассмотренных алмазоподобных фа3, LA10, может быть получена только из графита $\mathrm{L}_{4-8}$ ABCD при достижении давления 32-40 ГПа. Значения давлений, при которых формируются изученные фазы, значительно меньше давлений, необходимых для получения других структурных разновидностей алмаза $[13,14]$. Все структурные переходы «графиты $\mathrm{L}_{4-8}$ $\rightarrow$ алмазоподобные фазы» являются фазовыми переходами первого рода, в результате которых выделяется энергия от 0.3 до 0.6 эВ/атом.

Благодарность/Acknowledgement. Грешняков В. А. благодарит РФФИ за финансовую поддержку исследования (проект № 16-33-00030 мол_а). Беленков Е. А. благодарит Фонд перспективных научных исследований ЧелГУ за финансовую поддержку проведенного исследования. 


\section{Литература/References}

1. A.R. Verma, P. Krishna. Polymorphism and polytypism in crystals. New York-London-Sydney: John Wiley \& Sons, Inc., 1966, $341 \mathrm{p}$.

2. E. A. Belenkov, V. A. Greshnyakov. New Carbon Materials. 28 (4), 273-282 (2013). DOI: 10.1016/S1872-5805 (13) $60081-5$

3. Yu. E. Kitaev, M.I. Aroyo, J.M. Perez-Mato. Phys. Rev. B. 75 (6), 064110 (2007). DOI: 10.1103/PhysRevB.75.064110

4. E. A. Belenkov, V.A. Greshnyakov. Physics of the Solid State. 57 (1), 205-212 (2015). DOI: $10.1134 /$ S1063783415010047 [Е.А. Беленков, В.А. Грешняков. Физика твердого тела. 57 (1), $192-199$ (2015)]

5. E. A.Belenkov, V.A.Greshnyakov. Phys.SolidState.58(10), $2145-2154$ (2016). DOI: 10.1134/S1063784216100133 [Е.А. Беленков, В.А. Грешняков. ФТТ. 58 (10), 2069-2078 (2016)]

6. D.S. Lisovenko, Yu. A. Baimova, L. Kh. Rysaeva, V. A. Gorodtsov, S. V. Dmitriev. Phys. Solid State. 59 (4), $820-828$ (2017). DOI: $10.1134 / S 106378341704014 \mathrm{X}$ [Д.С. Лисовенко, Ю.А. Баимова, Л.Х. Рысаева, В. А. Городцов, С. В. Дмитриев. ФТТ. 59 (4), 801 - 809 (2017)]
7. K.S. Grishakov, K.P. Katin, M.M. Maslov. Adv. Chem. Phys. 2016, 1862959 (2016). DOI: 10.1155/2016/1862959

8. A.E. Galashev, O.R. Rakhmanova. Phys. Usp. 57 (10) 970-989 (2014). DOI: 10.3367/UFNe.0184.201410c.1 045 [А.Е. Галашев, О.Р. Рахманова. УФН. 184 (10), $1045-1065$ (2014)]

9. J.A. Baimova, Bo Liu, S. V. Dmitriev, K. Zhou. Physica Status Solidi RRL. 8 (4), 336-340 (2014). DOI: 10.1002/pssr.201409063

10. V.A. Greshnyakov, E. A. Belenkov. Letters on Materials. 6 (3), $159-162$ (2016). DOI: 10.22226/2410-3535-2016 -3-159-162 [В.А. Грешняков, Е.А. Беленков. Письма о материалах. 6 (3), 159-162 (2016)]

11. J. P. Perdew, A. Zunger. Phys. Rev. B. 23 (10), 5048 - 5079 (1981). DOI: 10.1103/PhysRevB.23.5048

12. J.P. Perdew, K. Burke., M. Ernzerhof. Phys. Rev. Lett. 77, 3865 - 3868 (1996). DOI: 10.1103/PhysRevLett.77.3865.1 0062328

13. V.A. Greshnyakov, E. A. Belenkov. Tech. Phys. 61 (10), $1462-1466$ (2016). DOI: $10.1134 / \mathrm{S} 1063784216100133$ [В. А. Грешняков, Е. А. Беленков. ЖТФ. 86 (10), 20 - 24 (2016)]

14. V. A. Greshnyakov, E. A. Belenkov. J. Exp. Theor. Phys. 124 (2), 265 - 274 (2017). DOI: 10.1134/S1063776117010125 [В.А. Грешняков, Е.А. Беленков. ЖЭТФ. 151 (2), $310-321(2017)]$ 